\title{
Towards molecular motors in unison
}

\author{
Built-in molecular motors in a porous crystal show unidirectional rotary motion fuelled by light.
}

\author{
Hubiao Huang and Takuzo Aida
}

iving organisms have evolved exquisite biological machinery to harness nanoscopic motion and translate it into macroscopic-scale motion. For example, chaperonin proteins release properly folded proteins from their cavity by triggering different interlocked movable parts; unicellular organisms realize directional locomotion by means of the collective swing of flagella; and some mammalian epithelia harbour multiple motile cilia to aid directional fluid flow ${ }^{1,2}$. Fascinated by such elaborate mechanisms, synthetic chemists have invented a number of artificial molecular machines to create molecules that are reminiscent of biological machinery. Although a wide variety of responsive molecules and materials that feature molecular motors and switches has been reported in the past few decades, it is still a challenge to densely pack and arrange movable units into a solid material to achieve a macroscopic task, without spoiling their intrinsic, nanoscopic dynamic properties ${ }^{2,3}$. Writing in Nature Nanotechnology, Danowski et al. ${ }^{4}$ now report a light-driven unidirectional rotary motion of built-in molecular motors within a rigid metal-organic framework (MOF) ${ }^{5}$.

The researchers synthesize a bispyridine ligand that has a chiral overcrowded alkene and succeed in immobilizing this rotary motor unit in a rigid pillared-layer MOF via a mild post-synthesis method: solvent-assisted ligand exchange ${ }^{6}$. The resulting MOF crystal accommodates in its framework about $10^{20}$ light-driven molecular motors per cubic centimetre in a spatial-specific and periodic manner. Moreover, the researchers show that each motor is able to make a full $360^{\circ}$ rotation within the voids of the nanoporous structure (Fig. 1a). On exposure of the MOF crystal to ultraviolet light (395 $\mathrm{nm}$ ), the built-in motor units start to rotate, all in the same direction, by means of two cycles of stepwise photochemical isomerization and thermally driven helix inversion. Interestingly, the rate constant and activation barrier for the motor units to rotate in the metalcoordination network are comparable to those in solution. These observations

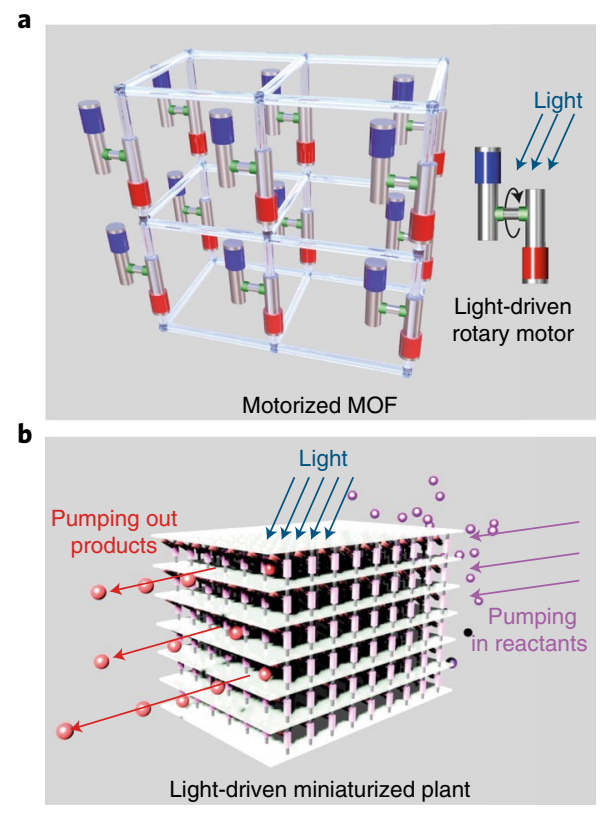

Fig. 1 | Motorized MOF. a, Periodic and tight arrangement of light-driven molecular motors in a porous pillared-layer crystalline framework, in which the built-in molecular motor units (red and blue structures) show unidirectional rotary motion at a rate comparable to that in solution. b, Possible light-driven MOF with a catalytic centre as a miniaturized future chemical plant, in which collective motions of the rotary motor units might accelerate the mass-transfer processes and therefore enhance the efficacy of production.

indicate that the uniform spatial environment of the porous coordination network enables even densely packed molecular motors to avoid intermolecular interactions with their neighbours ${ }^{7}$.

We envisage that this motorized MOF could serve as a light-powered pump to accelerate the flow of gases in nanochannels. Moreover, if a catalytic function were to be incorporated in their structure, one might be able to pump reactants and products in and out by exposure to ultraviolet light, and make a miniaturized chemical plant (Fig. 1b). To make this work, however, one needs to consider some practical issues such as the limited penetration depth of ultraviolet light and insufficient mechanical robustness of MOFs. In addition, other questions may arise: for example, when the MOF channels are tightly filled with gaseous molecules, do all the built-in motor units work synchronously? In other words, if the motor units located on one side of a particle rotate by selective photoirradiation, can the motor units on the other dark side rotate autonomously via the unidirectional flow of gaseous molecules? Furthermore, if the built-in chiral molecular motors are racemic, can the flow of gaseous molecules be accelerated by photoirradiation? At this point, all these questions are left for further exploration.

In general, we believe that to realize a higher level of sophistication, future work should address the issue of how to combine biological and artificial molecular machines in a spatially organized and interactive manner ${ }^{8}$. The design and understanding of the complementary role of these two components would be instrumental to the conceptual expansion of molecular machines into the realm of molecular intelligence. The promise is to inform topical technologies such as robotics with concepts developing at the border between chemistry, biology and nanoscience.

\section{Hubiao Huang ${ }^{1 \star}$ and Takuzo Aida ${ }^{1,2 \star}$ ${ }^{1}$ Department of Chemistry and Biotechnology, School of Engineering, The University of Tokyo, Tokyo, Japan. ${ }^{2}$ RIKEN Center for Emergent Matter Science, Saitama, Japan. \\ *e-mail: huang@macro.t.u-tokyo.ac.jp; aida@macro.t.u-tokyo.ac.jp}

Published online: 18 March 2019 https://doi.org/10.1038/s41565-019-0414-1

\footnotetext{
References

1. Zhou, F. \& Roy, S. Cell 162, 224-224 (2015).

2. Kinbara, K. \& Aida, T. Chem. Rev. 105, 1377-1400 (2005).

3. Feringa, B. L. Angew. Chem. Int. Ed. 56, 11060-11078 (2017).

4. Danowski, W. et al. Nat. Nanotechnol. https://doi.org/10.1038/ s41565-019-0401-6 (2019).

5. Yaghi, O. M. Nature 423, 705-714 (2003).

6. Karagiaridi, O., Bury, W., Mondloch, J. E., Hupp, J. T. \& Farha, O. K. Angew. Chem. Int. Ed. 53, 4530-4540 (2014).

7. Chen, K.-Y. et al. J. Am. Chem. Soc. 136, 3219-3224 (2014).

8. Sim, S. \& Aida, T. Acc. Chem. Res. 50, 492-497 (2017).
} 